\title{
Evaluation des Coupes-Rases Par la Télédétection et Les Systèmes d'Information Géographique dans la Forêt Classée de Djigbé (Bénin)
}

\author{
Eugène V. S. Gnonlonfin, \\ Adi Mama,
}

Laboratoire d'Ecologie Appliqué, Ecole d'Aménagement et de Gestion de

l'Environnement, Faculté des Sciences agronomiques,

Université d'Abomey-Calavi (UAC), Bénin, Cotonou

\section{Faustin Y. Assongba,}

Ecole Nationale Supérieure des biosciences et Biotechnologies Appliquées, Université Nationale des Sciences et Technologies Ingénierie et

Mathématiques (UNSTIM) d'Abomey, Bénin

\section{Julien G. Djego,}

Laboratoire d'Ecologie Appliqué, Ecole d'Aménagement et de Gestion de

l'Environnement, Faculté des Sciences agronomiques,

Université d'Abomey-Calavi (UAC), Bénin, Cotonou

Doi:10.19044/esj.2020.v16n21p310 URL:http://dx.doi.org/10.19044/esj.2020.v16n21p310

\section{Résumé}

Le paysage des forêts classées étant de plus en plus anthropisé à cause de la pression démographique, optimiser le suivi du prélèvement du bois serait un défi pour la gestion rationnelle des forêts au sud Bénin. A cet effet, cette étude a pour objectif d'évaluer l'exploitation forestière faite par coupes-rases. Ce qui implique une estimation de l'évolution du massif forestier et donc de la disponibilité en bois pour une gestion planifiée et durable de la forêt classée de Djigbé (commune de Zè). La méthode utilisée relève de la télédétection et les Systèmes d'Information Géographique (SIG). Elle inclue la photo interprétation par classification supervisée, le calcul de l'indice normalisée de végétation (NDVI) aboutissant à la restitution cartographique des coupesrases. Ainsi, la détection des coupes-rases a été suivie à partir des images satellitaires de type Landsat 8 des années 2014 à 2017. La détection automatisée des changements d'un état végétatif à un état non végétatif basée sur l'analyse de l'indice normalisé de la végétation (NDVI) entre deux dates a été utilisée. Des missions de géoréférencement des zones de coupes-rases dans la forêt classée de Djigbé ont aidé aux traitements des images à partir du logiciel ENVI 4.7. Les coupes-rases détectées ont par la suite été restituées par 
cartographie. Les résultats de détection des coupes-rases ont été statistiquement vérifiés par des matrices de confusion. Les emplacements de coupes-rases détectées pendant la période Décembre 2014-décembre 2015 diffèrent de celle de décembre 2015-janvier 2017. Chaque classe de coupe regroupée suivant un degré de certitude, respectant donc un cloisonnement donné. Ce qui renseigne sur la précision de la méthode automatisée de détection. La détection des coupes a été réalisée suivant trois valeurs de probabilité de présence de coupes-rases. Ces valeurs de probabilité sont : faible $(1 \sigma)$, moyenne $(2 \sigma)$ et forte $(3 \sigma)$ désignées par l'expression « degré de certitude ». Quelle que soit la classe et la période, le nombre de coupe est plus important chronologiquement pour le degré de certitude faible $(1 \sigma)$, moyenne $(2 \sigma)$ et forte $(3 \sigma)$. Les coupes-rases de détection supérieure ou égale à 1 ha dans la plantation domaniale de Djigbé couvrent 234,63 ha. Ce qui induit une estimation de la disponibilité en bois qui est diminuée de cette même superficie détectée durant la période prise en compte par l'étude. Considérant le classement des coupes-rases suivant les degrés de certitude «moyenne » et " forte ", sur l'ensemble des 234,63 ha, les proportions détectées ont été respectivement de 76 et de $24 \%$. La détection par images satellitaires et la cartographie est un outil de suivi et d'évaluation pertinent. Cet outil contribue à l'analyse périodique d'images satellitaires et la simulation de l'évolution de la ressource bois en plantation. Les résultats de cette étude permettent ainsi d'apprécier avec impartialité l'évolution du prélèvement et le suivi à distance de l'exploitation du bois. L'appropriation de l'utilisation de cet outil par les acteurs forestiers pour l'estimation de la ressource bois disponible, contribuerait à une meilleure gestion des forêts.

Mots-clés : Systèmes d'Information Géographique, plantation, télédétection, coupes-rases, exploitation forestière, Djigbé 


\title{
Clear-Cuts and Wood Availability Assessment using Remote Sensing and Geographic Information System at the State Forest of Djigbé in Benin
}

\author{
Eugène V. S. Gnonlonfin, \\ Adi Mama,
}

Laboratoire d'Ecologie Appliqué, Ecole d'Aménagement et de Gestion de

l'Environnement, Faculté des Sciences agronomiques,

Université d'Abomey-Calavi (UAC), Bénin, Cotonou

\section{Faustin Y. Assongba,}

Ecole Nationale Supérieure des biosciences et Biotechnologies Appliquées, Université Nationale des Sciences et Technologies Ingénierie et

Mathématiques (UNSTIM) d'Abomey, Bénin

\section{Julien G. Djego,}

Laboratoire d'Ecologie Appliqué, Ecole d'Aménagement et de Gestion de

l'Environnement, Faculté des Sciences agronomiques,

Université d'Abomey-Calavi (UAC), Bénin, Cotonou

\section{Abstract}

The landscape of classified forests us being more and more anthropized because of demographic pressure. As a result, optimizing the monitoring of wood removal would be a challenge for the rational management of forests in southern Benin. This paper focuses on assessing logging done by clear-cutting. This implies an estimate of the evolution of the forest massif and therefore of the availability of wood for a planned and sustainable management of the classified forest of Djigbé (commune of Zè). The method used is based on remote sensing and Geographic Information Systems (GIS). It includes photo interpretation by supervised classification and the calculation of the standardized vegetation index (NDVI) leading to the cartographic restitution of the clear-cuts. Thus, the clearing detection was followed from Landsat 8 type satellite images from 2014 to 2017. Automated detection of changes from a vegetative to a non-vegetative state based on the analysis of the index normalized vegetation (NDVI) between two dates was used. Georeferencing missions of clearcut areas in the classified forest of Djigbé helped image processing using the ENVI 4.7 software. The level cuts detected were subsequently restored by mapping. The shaving cutter detection results were statistically verified by confusion matrices. The clearing locations detected 
during the period December 2014 to December 2015 differ from that of December 2015 to January 2017. Each cutting class was grouped according to a degree of certainty, thus respecting a given partitioning. This provides information on the accuracy of the automated detection method. The detection of the cuts was carried out according to three values of probability of the presence of clear-cuts. These probability values are: low $(1 \sigma)$, medium $(2 \sigma)$, and high $(3 \sigma)$ designated by the expression "degree of certainty". Whatever the class and the period, the number of cuts is more important chronologically for the degree of certainty low $(1 \sigma)$, medium $(2 \sigma)$, and strong $(3 \sigma)$. The leveling shafts of detection greater than or equal to 1 ha in the national plantation of Djigbé cover 234.63 ha. This leads to an estimate of the availability of wood, which is reduced by the same area detected during the period taken into account by the study. Considering the classification of clearcuts according to the degrees of certainty "medium" and "strong", on the whole of the 234.63 ha, the proportions detected were respectively 76 and $24 \%$. Detection by satellite images and mapping is a relevant monitoring and evaluation tool. This tool contributes to the periodic analysis of satellite images and the simulation of the evolution of the wood resource in plantation. The results of this study thus allow an impartial assessment of the evolution of the harvest and remote monitoring of logging. The appropriation of the use of this tool by forest stakeholders, for the estimation of the available wood resource, would contribute to better forest management.

Keywords: Systèmes d'Information Géographique, plantation, télédétection, coupes-rases, exploitation forestière, Djigbé

\section{Introduction}

La dégradation des forêts est devenue un problème grave, en particulier dans les pays en développement (OIBT, 2005). Au Bénin, l'exploitation et la commercialisation du bois constituent une des importantes menaces de la biodiversité forestière (DGFRN, 2010). Avec les plantations fournissant d'énormes quantités de bois et vu l'amenuisement des formations naturelles de par leur exploitation (Mama, 2013), cette pression s'oriente dorénavant sur les plantations. Certaines espèces forestières sont plus prisées par les exploitants. En l'occurrence, le bois du teck est un produit très recherché entrainant une surexploitation des plantations existantes (Akouehou \& Ayelo, 2005). Il revêt une grande importance socioéconomique. Face à ce problème, il avait été déjà annoncé en 2005 que des initiatives techniques sont nécessaires pour gérer durablement les peuplements de Teck (Akouehou \& Ayelo, 2005). La forêt classée de Djigbé, une plantation essentiellement de teck est l'une des plus grandes réserves de bois au Sud-Bénin en dehors de la forêt classée de la Lama et a été mise en 
place par l'Office Nationale du Bois du Bénin (ONAB). Même si son mode de gestion participative adoptée vise à assurer une protection durable en partageant les intérêts de celle-ci avec les riverains, ceci nécessite un suivi optimal du fait de coupes frauduleuses existantes (ONAB, 2005). Ces coupes frauduleuses dans les plantations, pratiquées par les populations riveraines, font partie des facteurs de dégradation des plantations forestières (ONAB, 2005). A Djigbé, selon la même source, ces coupes illicites sont inventoriées à 931,5 ha. Ce qui mérite une attention particulière des gestionnaires en matière d'intensification des mesures de protection. Le contrôle de l'exploitation est une étape essentielle d'une exploitation forestière durable (Gond \& Guitet, 2009). A l'heure actuelle, l'exploitation mise en œuvre est de type conventionnel. Ainsi, la rareté de données statistiques fiables sur le potentiel forestier actuel, rend difficile la connaissance de la production annuelle de bois constituant une contrainte au développement du secteur au Bénin (Quenum, 2005). Or comme le mentionne FAO (2003), le manque de ressources humaines pour la gestion des forêts secondaires est manifeste au Bénin malgré les dispositions institutionnelles. Dans un contexte de manque de ressources humaines en particulier dans le domaine forestier, l'outil télédétection et les SIG sont un complément important pour le suivi du prélèvement et de l'évolution du massif forestier. La télédétection a déjà fait ses preuves dans la distinction les espaces forestières exploitées des autres et permet d'appréhender l'impact de l'exploitation forestière sur la forêt à partir d'imagerie satellitaires (Souza et al., 2003 ; Furusawa et al., 2004 ; Gond \& Guitet, 2009 ; Read et al., 2013). Plusieurs travaux d'aménagement et de recherche ont été entrepris sur les teckeraies domaniales du Bénin. Certains ont mis en évidence les relations entre les facteurs écologiques, la végétation du sous-bois et la productivité des teckeraies. Celles-ci permettant d'identifier et de cartographier plusieurs stations forestières (Noumon \& Ganglo, 2005 ; Dossa et al., 2005 ; Dossa \& Ganglo, 2006 ; Aoudji \& Ganglo, 2006; Aoudji et al., 2006 ; Ganglo \& Foucault, 2006 ; Noumon et al., 2006 ; Tohngodo et al., 2006a,b ; Yêvidé et al., 2011 ; Toyi, 2013). Ces travaux ne tiennent pas compte du suivi de l'état de prélèvement des plantations utilisant une approche d'évaluation par la télédétection et les SIG. Or Cette démarche est économiquement acceptable pour une observation rapide et complète sur un territoire étendu (Cheret et al., 2011). C'est la meilleure méthode (rapport qualité-coût) pour cartographier les ressources forestières (Renaud et al., 2015). A travers les images satellitaires, une aide significative pour la connaissance de la couverture végétale et le suivi de son état est possible (Cheret et al., 2011). Pour la forêt, la télédétection permet le suivi de la dégradation et du déboisement à l'échelle régionale. Localement, l'étude des coupes-rases en milieu forestier est également possible (Crutzen, 2017). La «coupe-rase» désignant, en sylviculture, un mode d'aménagement sylvicole 
passant par l'abattage de la totalité des arbres d'une parcelle ou d'une exploitation forestière. C'est dans un souci de rationalisation de cette ressource rare, que la télédétection satellitaire pourrait contribuer à l'évaluation des coupes-rases dans la forêt classée de Djigbé. Des applications ont montré l'intérêt pour l'évaluation du dépérissement forestier dans un contexte de modification brutale et importante de l'activité photosynthétique (Deshayes et al., 2006; Solberg et al., 2007). Les indices de végétation ont été largement utilisés pour la surveillance des conditions de végétation (Tchibozo \& Toundoh, 2014). Dans cet article, l'indice normalisé de végétation calculé à partir des images satellites sera utilisé pour localiser et cartographier les zones de végétation prélevées en terme de coupes-rases.

\section{Milieu d'étude}

La présente étude a été conduite dans la plantation domaniale du secteur forestier de Djigbé. Elle est comprise entre les latitudes $6^{\circ} 81^{\prime} 75^{\prime \prime}$;

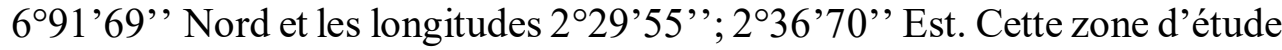
est située au Sud du Bénin, dans le département de l'Atlantique, couvrant en grande partie la Commune de Zè et dont une infime portion s'étend sur la Commune de Toffo. Cette zone bénéficie d'un climat de type subéquatorial caractérisé par une pluviométrie moyenne annuelle d'environ $1100 \mathrm{~mm}$. Elle est caractérisée par quatre saisons: deux saisons pluvieuses alternant avec deux saisons sèches. Ces conditions climatiques sont favorables au développement de la sylviculture générale et celle du teck en particulier de part ses exigences écologiques (Toyi, 2012). La plantation domaniale du secteur forestier de Djigbé est de type industriel. L'essentiel de sa superficie est affectée aux plantations de teck pour la production de bois d'œuvre (MEPN et PNUD, 2009). Les rémanents sont utilisés comme bois de chauffage par les riverains qui en exportent aussi dans les centres urbains. La même espèce (teck) est la plus plantée par les promoteurs privés de plantation de la commune. La sylviculture à petite échelle (plantations privées) est la principale pourvoyeuse de bois de service dans la région, grâce aux perches de teck (Toyi, 2012). 

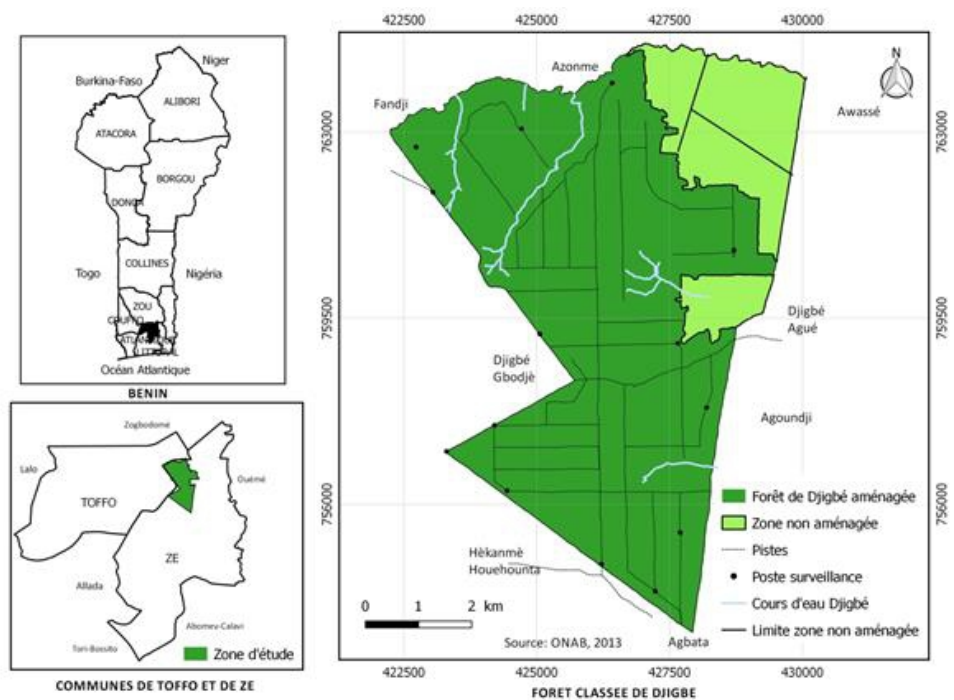

Figure 1. Localisation géographique de la zone d'étude (Forêt classée de Djigbé) Source : ONAB, 2013 et fond topographique IGN

\section{Matériel et méthodes de détection des coupes-rases}

\section{Matériel}

\section{Matériel d'étude}

Le matériel d'étude est la plantation forestière industrielle domaniale. L'espèce végétale ciblée par l'étude et présente essentiellement est le teck (Tectona grandis). Elle couvre 92, 32\% de superficie dans la forêt classée de Djigbé (ONAB, 2005).

Les outils utilisés pour la collecte de données sont :

- un GPS (modèle GPSMAP 60CSx) pour le levé d'informations concernant l'orientation, l'identification des parcelles par la prise des coordonnées de référence, le tracé des parcelles et la vérification des classifications;

- une machette pour ouvrir des pistes et dégager les pourtours des plantations avant la mesure des surfaces;

- un stylo, un crayon et une gomme utilisés pour la prise de notes pendant les enquêtes.

- des images satellitaires;

- $\quad$ une carte d'aménagement et de parcellisation en tant que document de base ayant servi à la circonscription et à une meilleure connaissance de la zone étudiée.

\section{Population d'étude}

La plantation domaniale de Djigbé a été choisie d'abord du fait qu'au Sud du Bénin, elle est une des grandes plantations domaniales et classée essentiellement constituée de teck. Le bois de cette espèce étant un produit très 
recherché en raison de sa valeur et son intérêt économique. Cette situation entraîne une surexploitation des plantations existantes (Akouehou \& Ayelo, 2005). C'est un vaste couvert végétal. Elle est en outre bien aménagée, parcellisée de sorte qu'on identifie clairement des zones qui ont subi des mutations d'un sol couvert à un sol nu sous l'effet des coupes grâce à l'imagerie satellitaire. Ceci évite des contrastes de détection automatisée par les images satellitaires utilisées.

L'échantillon étudié est constitué des parcelles de plantations de teck coupées entre 2014 et 2017 dans la forêt de Djigbé. La technique d'échantillonnage est basée sur des documents cartographiques reçus de l'inspection forestière et facilitant l'identification des périodes de coupes. Des parcelles de coupe rases géoréférencées pendant les travaux de terrain ont été aussi utiles à l'échantillonnage. Ces parcelles ont été systématiquement recensées. Sur les 4000 ha de superficies morcelées en 204 parcelles, 15 parcelles de coupes-rases ont été identifiées. A partir de ces 15 parcelles, 100 points de références ont été défini sur image satellitaire lors de l'opération de détection automatisée. Ces 100 points de références définis sont largement supérieur au minimum 50 points que recommande la littérature scientifique. Cela assure un équilibre entre la représentativité statistique et le temps de photo-interprétation (Ose \& Deshayes, 2015). Les données de référence ont donc découlé des documents géoréférencés et des parcelles géoréférencées sur le terrain.

\section{Méthodes de collecte des données}

Les données collectées pour détecter les coupes-rases et leur dynamique sont multisources. Il s'agit :

- des images satellitaires Landsat de résolution $30 \mathrm{~m}$ : en décembre 2014, en décembre 2015 et en janvier 2017. Le faible écart d'un mois observé à la deuxième période se justifie par un souci d'utiliser des images ayant moins de bruits.

- Des prises de coordonnées géographiques des parcelles coupées. Des parcelles de plantation et d'autres types d'occupation de sol (espaces de sol non dus aux coupes, des campements, des parcelles de cultures) ont été géoréférencés par des prises de points fixes et par tracking.

- des entretiens semi-structurés avec l'inspection forestière nous a fourni des données de références pour la période 2014-2015.

- des missions de géo-référencement des zones de coupes-rases dans la forêt classée de Djigbé ont été faites avec l'aide d'un guide qui nous a renseigné et conduit sur les sites de coupes visibles de l'année 2017. L'observation de ces coupes récentes, a permis d'avoir les références de la période 2015-2017. Les données de référence ont été utiles pour 
le choix des types d'occupation (coupe- rases et plantation) lors des classifications d'image. Celles collectées sur le terrain ont surtout servies à l'évaluation statistique avec le logiciel QGIS. Les données préexistantes collectées, ont été évaluées par traitement d'image satellitaire avec le logiciel ENVI (classification supervisée). Les parcelles examinées ont été ensuite comparées aux résultats de la classification.

\section{Méthodes de détection automatisée des coupes-rases}

La détection par images satellitaires est un outil de suivi et d'évaluation performant pour une gestion durable des grandes plantations ou forêts. La méthodologie utilisée à cet effet est celle développée par Ose et Deshayes-Irstea (2015). Elle repose sur la détection automatisée des changements d'un état végétatif boisé à un état de sol-nu à partir de deux images satellitaires, acquises entre deux dates. Le principe de la détection est l'analyse de l'évolution de l'indice NDVI entre deux dates. La procédure d'extraction a été scindée en deux grandes étapes :

- Une phase de prétraitement ou de mise en conformité des données pour assurer la comparabilité en termes de géométrie et de radiométrie,

- Une phase de détection et de classification des changements pour aboutir à la cartographie des coupes-rases.

\section{Prétraitement des données satellitaires : Calibration en réflectance top of atmosphere (TOA)}

Il s'agit d'abord d'obtenir des fichiers images géométriquement superposables et restitués dans un référentiel connu. Les valeurs de pixels (comptes numériques) ont été transformées en réflectance dite TOA (Top Of Atmosphere). Il a été donc procédé à la calibration en réflectance.

Il a été utilisé une extension QGIS, développée par Irstea/Geosud qui permet de convertir automatiquement en réflectance TOA les images issues de certains capteurs dont Landsat 8. L'objectif de son choix est d'éviter des erreurs de manipulations et de gagner en temps de traitements.

En prélude aux opérations de traitement d'images, un masque a été appliqué au cours du prétraitement des données satellitaires. Ceci afin de ressortir les informations qui concernent exclusivement les secteurs (parcelles) cibles. Etant donné que les images utilisées couvrent une portion plus importante. 


\section{Détection des changements et extraction des coupes-rases Détection des changements par différence des NDVI}

Pour détecter les changements, les indices normalisés de végétation ont d'abord été calculés pour chaque image. Ils se calculent d'après la formule suivante :

$$
\begin{gathered}
N D V I=\left(\begin{array} { c } 
{ \rho _ { - } T O A ^ { \wedge } P I R - \rho _ { - } T O A ^ { \wedge } \text { Rouge } ) } \\
{ + \rho _ { - } T O A ^ { \wedge } \text { Rouge } ) }
\end{array} \left(\rho_{-} T O A^{\wedge} P I R\right.\right. \\
\end{gathered}
$$

Où $\rho_{T O A}^{P I R}$ est la réflectance de la bande proche infrarouge et $\rho_{\text {TOA }}^{\text {Rouge }}$ celle de la bande rouge. Le NDVI étant compris entre -1 et 1 .

Ensuite, une opération arithmétique de soustraction a été appliquée aux images issues du calcul des NDVI. Cette approche a l'avantage de traiter des données normalisées, afin de faciliter les comparaisons dans le temps.

La différence des NDVI a été calculée pour chacune des deux années permettant de détecter des changements. Ses valeurs sont comprises entre -2 et 2. $\Delta_{N D V I}=N D V I_{D 2}-N D V I_{D 1}$ où $N D V I_{D 1}$ et $N D V I_{D 2}$ sont respectivement les indices normalisés de végétation des années $n$ et $n+1$. Les images Landsat 8 du 15 décembre 2015 et Landsat 8 du 18 janvier 2017 correspondant respectivement aux dates D1 et D2 ont servi aux opérations de traitement. La différence entre les deux néocanaux $\Delta_{N D V I}\left(N D V I_{D 2}-\right.$ $\left.N D V I_{D 1}\right)$ a permis d'identifier trois types d'évolutions: les parcelles ayant peu ou pas changées ont des valeurs voisines de 0 . Les zones qui sont passées de sol nu à la végétation ont des valeurs positives, significativement différentes de 0 , avec un maximum de 2 . Les zones qui sont passées de la végétation au sol nu ont des valeurs négatives, significativement différentes de 0 , avec un minimum de -2 .

\section{Classification des coupes-rases}

Les coupes-rases détectées ont été classifiées par « degré de certitude ». La notion «degré de certitude» est empruntée à Ose et Deshayes-Irstea (2015). Certains auteurs emploient d'autres notions de définition équivalente. Ainsi, pour Ferer (2015) c'est « degré de précision qui est exprimée dans son document. Alors que Lambert (2015) a employé la notion «classe de probabilité ». L'une ou l'autre renvoie à un regroupement en classes des résultats de détection automatisée des coupesrases selon la probabilité qu'une portion (zone/pixel) donnée soit affectée par une coupe-rase. Les classes sont ainsi regroupées par hiérarchie selon que le résultat de détection des coupes-rases conformément à la variation de la NDVI montre la probabilité de présence de coupes-rases. Trois degrés de certitude $1 \sigma, 2 \sigma$ et $3 \sigma$ ont été considérés représentant respectivement le degré de certitude faible, moyen et fort. Le choix de trois classes est fait dans l'optique d'affiner l'analyse de surface de coupes détectées par la technique 
automatisée afin d'apprécier son opérationnalité. Il peut être relevé de légers écarts dans la variation de NDVI. En effet, la plantation qui n'a pas changée peut cependant voir son NDVI varier légèrement, et son $\Delta_{N D V I}$ varie donc dans une plage de plus ou moins avec un écart-type autour de la moyenne. Les valeurs situées entre le minimum (-2) et la moyenne des valeurs de $\Delta_{N D V I}$ moins un écart-type traduisent alors le passage d'un sol boisé à l'année $\mathrm{n}$ à un sol nu ou peu végétalisé à l'année $\mathrm{n}+1$.

Les changements ont été identifiés au moyen de requêtes lancées via une calculatrice raster dans QGIS. La requête étant un langage informatique reconnu par la machine interrogeant une base de données. Elle permet d'obtenir des données vérifiant certaines conditions.

Requête $1=\left[-2<\Delta_{N D V I}<(m-\sigma)\right]$ (Ose \& Deshayes-Irstea, 2015) où $\mathrm{m}$ et $\sigma$ sont respectivement la moyenne et l'écart-type des valeurs des pixels sur l'image $\Delta_{N D V I}$. La requête 1 livre en sortie une image binaire dans laquelle les pixels positifs (codés1) correspondent aux zones supposées coupées et ceux négatifs (codés0) sont les zones supposées non-coupées.

Ce résultat reste peu précis et difficilement interprétable puisqu'il attribue une même valeur à différents types de coupes-rases : coupes récentes ou anciennes, coupes certaines ou probables. Il a donc été décomposé les valeurs comprises dans l'intervalle de la requête 1 en trois classes ordonnées suivant un degré de certitude. Ainsi, plus la valeur d'un pixel s'éloigne de la moyenne de $\Delta_{N D V I}$, plus forte est la NDVI; certitude que ce soit une coupe rase récente. L'écart-type dimensionne l'amplitude des classes. L'expression proposée par Ose et Deshayes-Irstea (2015) est exprimée comme suit

Requête 2 : degré1 $=\left[(m-2 * \sigma) \leq \Delta_{N D V I}<(m-\sigma)\right]$

degré2 $=\left[(m-3 * \sigma) \leq \Delta_{N D V I}<(m-2 * \sigma)\right]$

degré3 $=\left[-2<\Delta_{N D V I}<(m-3 * \sigma)\right]$

De cette requête, résulte des images codées en entiers compris entre 0 et 3 . La valeur 0 désignant toute partie de l'image boisée (sans coupesrases), les valeurs 1 à 3 correspondent aux détections de coupes-rases suivant respectivement un degré de certitude faible, moyen ou fort.

Chaque classe de coupe regroupée suivant un degré de certitude, respecte un cloisonnement donné. Ce qui renseigne sur la précision de la méthode automatisée de détection classant les coupes-rases en fonction de la variation des valeurs des différences de NDVI comprises entre -2 et +2 . Ainsi, la détection de la valeur d'un pixel plus proche de la moyenne de $\Delta \_$NDVI est classée véritablement par le logiciel comme une coupe de faible certitude (Figures 4 b et 5 b). Inversement, celles s'éloignant de la moyenne de $\Delta_{-}$NDVI sont des coupes-rases de forte certitude (Figures $4 \mathrm{~d}$ et $5 \mathrm{~d}$ ). 


\section{Evaluation statistique}

Afin de procéder à l'évaluation statistique des données, les images issues de la requête 2, ont d'abord été converties en vecteur au format «shapefile » (ESRI). Chaque entité a été récupérée et organisée selon son degré de certitude. La table attributaire en sortie est enrichie d'un nouveau champ nommé surface dans lequel sont calculées les superficies en hectares de chaque entité.

Ensuite, la qualité d'extraction des coupes-rases a été statistiquement évaluée de façon automatisée par la classification supervisée dans le logiciel ENVI 4.7. Les régions d'intérêt (ROI) choisis ont été vérifiées par les réalités observées sur le terrain pendant les travaux de collecte de données en décembre 2017. L'évaluation statistique proprement dite de la détection des coupes-rases de 2014-2015 a été faite à partir de l'image Landsat 8 (2015). Les résultats de cette évaluation ont été déduis au moyen d'une matrice de confusion. Chaque colonne de la matrice représente le nombre d'occurrences d'une classe estimée, tandis que chaque ligne représente le nombre d'occurrence de la référence. Les parcelles examinées ont été ensuite comparées aux résultats de la classification. À partir de cette matrice, ont été calculées: la précision globale, la précision pour l'utilisateur, la précision pour le producteur, les erreurs d'omission et de commission et le coefficient de Kappa.

Les erreurs d'omission et de commission sont calculées par les formules ciaprès (Lambert, 2014):

$$
\begin{gathered}
\text { Erreur d'omission }(\%)=\frac{\text { Pixels coupés classés comme non }- \text { coupés }}{\text { Total des pixels coupés de références }} \\
\text { Erreur de commission }(\%) \\
=\frac{\text { Pixels non }- \text { coupés classés comme coupés }}{\text { Total des pixels coupés de référence }}
\end{gathered}
$$

\section{Résultats}

Détection par télédétection satellitaire et cartographie des coupes-rases Répartition des coupes-rases détectées par degré de certitude

Les coupes-rases détectées en fonction du degré de certitude et de classes de surfaces de 2014 à 2017 sont présentées sur la Figure 2. Seules les coupes-rases de plus 0,09 ha $\left(900 \mathrm{~m}^{2}\right)$ sont considérées par l'outil de télédétection du fait de la résolution spatiale des images utilisées. Il a été retenu les surfaces de coupes-rases supérieures ou égales à 0,5 ha. Quelle que soit la classe et la période, le nombre de coupe est plus important respectivement pour le degré $1 \sigma, 2 \sigma$ et $3 \sigma$. Plus le degré de certitude est faible, plus le nombre de détection est important et susceptible de prendre en compte de fausses détections. Par contre, plus la certitude est forte, moins nombreux sont les coupes-rases détectées. Dans ce cas, presque uniquement 
les coupes-rases sont détectées. En conséquence, plus le degré de certitude est fort, plus la détection est tamisée optimisant le résultat de la détection.

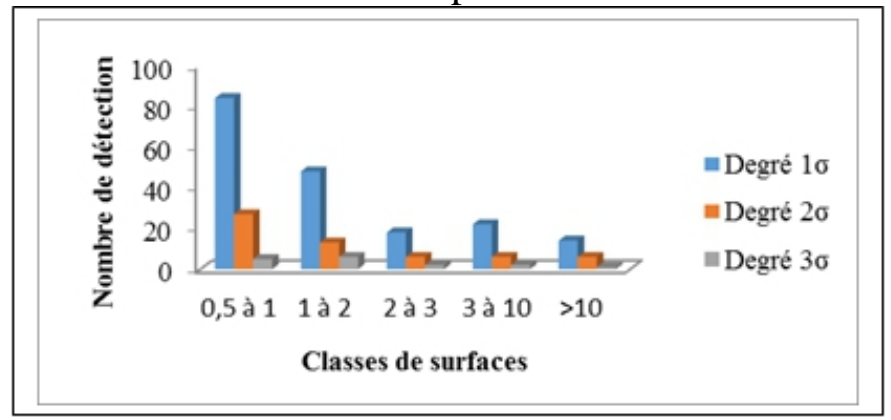

Figure 2. Détection des coupes-rases en fonction des classes de surfaces et du seuil de détection, 2014-2015-2017.

Distribution des coupes-rases de faible, moyenne et forte certitude de 2014 à 2015 et de 2015 à 2017

Les coupes rases détectées sur une portion de la zone d'étude sont présentées sur les Figures 3 et 4 . La même portion de chaque image est présentée pour distinguer nettement les coupes quel que soit leur degré de certitude. Chaque portion a été éclatée suivant les degrés de faible certitude ( 3 b et 4 b), de moyenne certitude ( 3 c et 4 c) et de forte certitude ( $3 \mathrm{~d}$ et $4 \mathrm{~d}$ ) pendant les périodes 2014-2015 et 2015-2017.

Les polygones en couleur beige correspondent à la détection des coupes-rases de degrél $\sigma$ soit pour des valeurs de différence de NDVI comprises entre le minimum et $(\mu-\sigma)$. Les polygones en couleur orange correspondent à la détection des coupes-rases de degré $2 \sigma$, soit pour des valeurs de différence de NDVI comprises entre le minimum et $(\mu-2 \sigma)$. Les polygones en couleur rouge correspondent à la détection des coupes-rases de degré $3 \sigma$ soit pour des valeurs de différence de NDVI comprises entre le minimum et $(\mu-3 \sigma)$. 


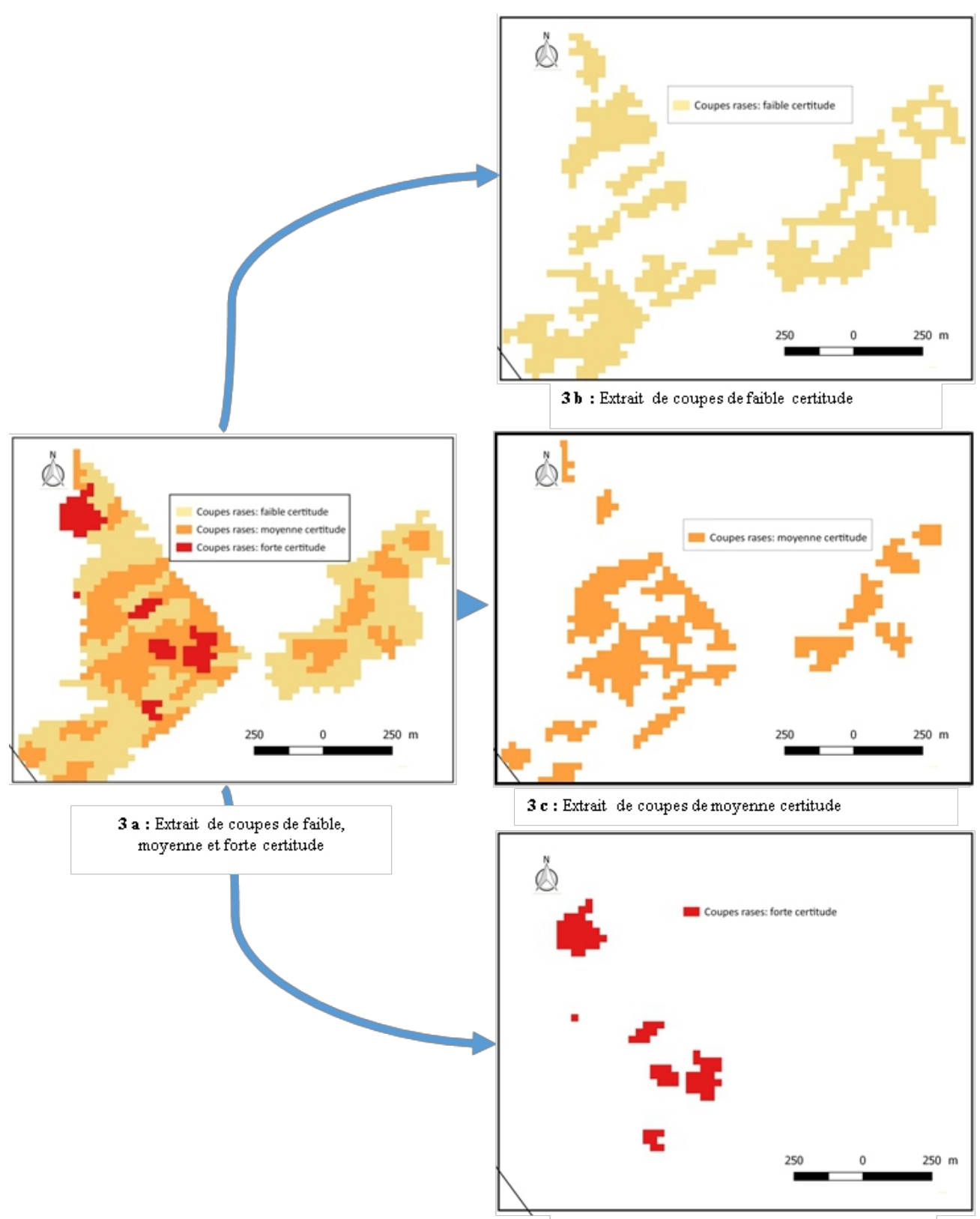

3d : Extrait de coupes de forte certitude

Figure 3 : Extrait de répartition des coupes-rases par degré de certitude, 20142015 


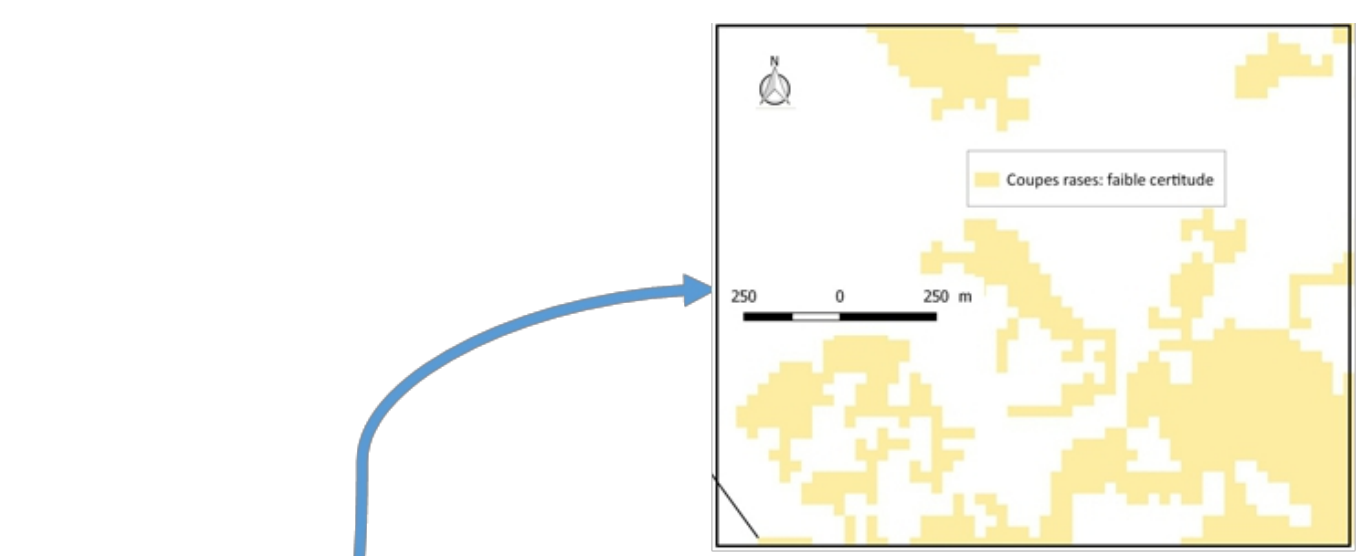

$4 \mathbf{b}$ : Extrait de coupes de faible certitude

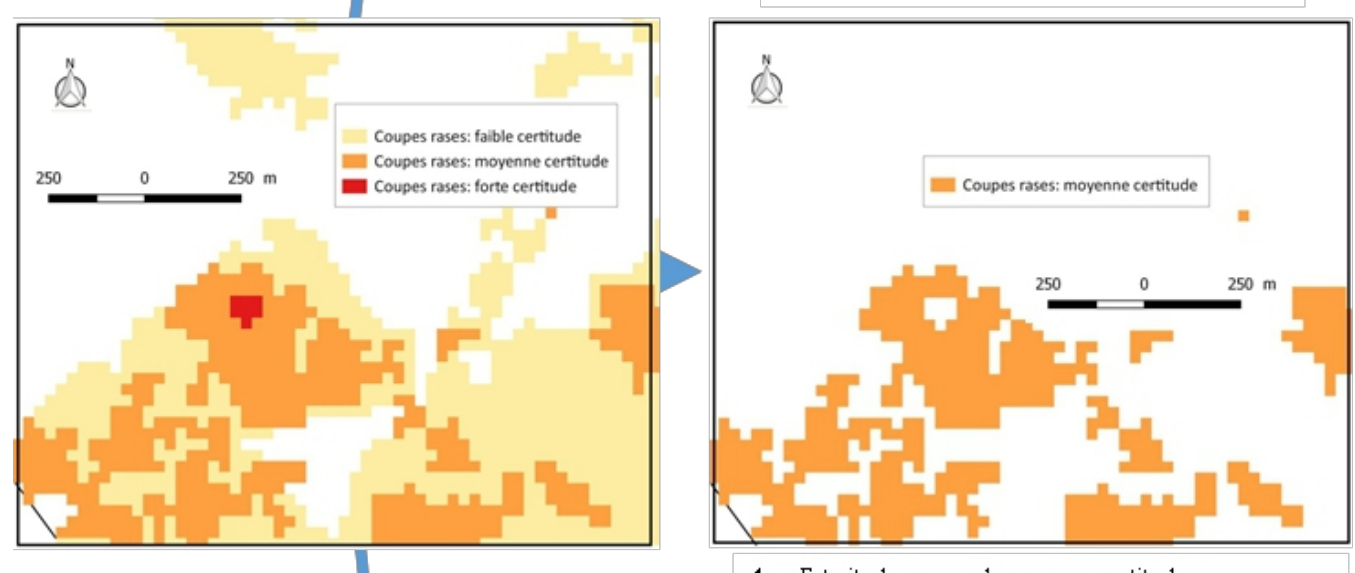

$4 \mathrm{c}:$ Extrait de coupes de moyenne certitude

4 a : Extrait de coupes de faible, moyenne et forte certitude
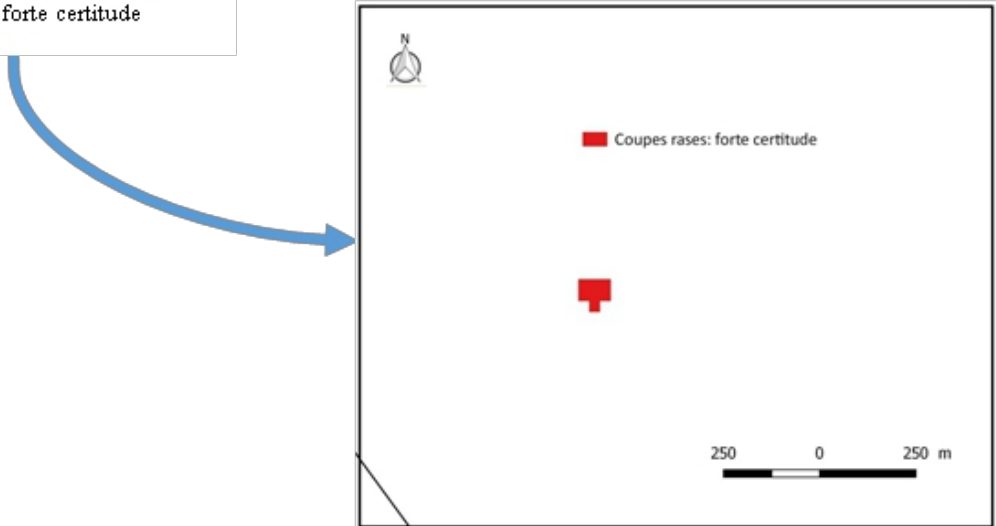

$4 \mathbf{d}:$ Extrait de coupes de forte certitude

Figure 4.Extrait de répartition des coupes-rases par degré de certitude, 2015-2017 


\section{Analyse de distribution des coupes-rases pour les périodes de 2014-2015 et 2015-2017}

Deux périodes de changements d'état végétatif boisé sont présentées à la Figure 5. Les détections ayant des formes allongées (désignées par des cadrans ovales rouges) apparaissant comme des effets de lisière et ne s'observent que sur les coupes-rases de faible certitude. Aucun pixel localisé coupe-rase pendant la période 2014-2015 n'est identifié à nouveau comme tel pendant la période 2015-2017. Comme le montre les flèches bleues. Ceci exclut toute confusion de détection pour les deux périodes de changement de l'état végétatif à l'état de coupe. Les plus grandes surfaces observées pour le degré de faible certitude sont d'éventuelles fausses détections. Ce qui peut s'expliquer selon nos investigations sur le terrain, par la confusion avec les changements d'état végétatifs au sol nu des zones de cultures. Car, il arrive qu'après les coupes-rases et l'enlèvement des grumes, l'ONAB (Office Nationale de Bois) autorise le COGEPAF (Comité de Gestion Participative de la Forêt) à enlever des rémanents au profit des populations riveraines. Ces villageois entretiennent certaines superficies de bandes coupées en y produisant des cultures vivrières avant que des jeunes plants soient remis en terre pour une nouvelle plantation.

Les coupes-rases de faible degré sont abondantes et dispersées. A ce degré, la détection peut être moins précise. Ceci peut s'expliquer par la coupe des plantations en bandes discontinues de manière à ce que deux bandes coupées intercalent une bande non coupée ou vis-versa. Or, les bandes sont souvent de largeur inférieure à la résolution spatiale de l'image Landsat 8 . A ce seuil, la détection est donc peu précise. Cette méthode de coupes-rases dans la plantation de Djigbé peut donc impacter la précision de la détection automatique utilisant une image satellitaire Landsat 8 si on se limite à ce degré de précision. L'acquisition et l'utilisation d'images de haute résolution donnerait de meilleurs résultats dans ce contexte. 


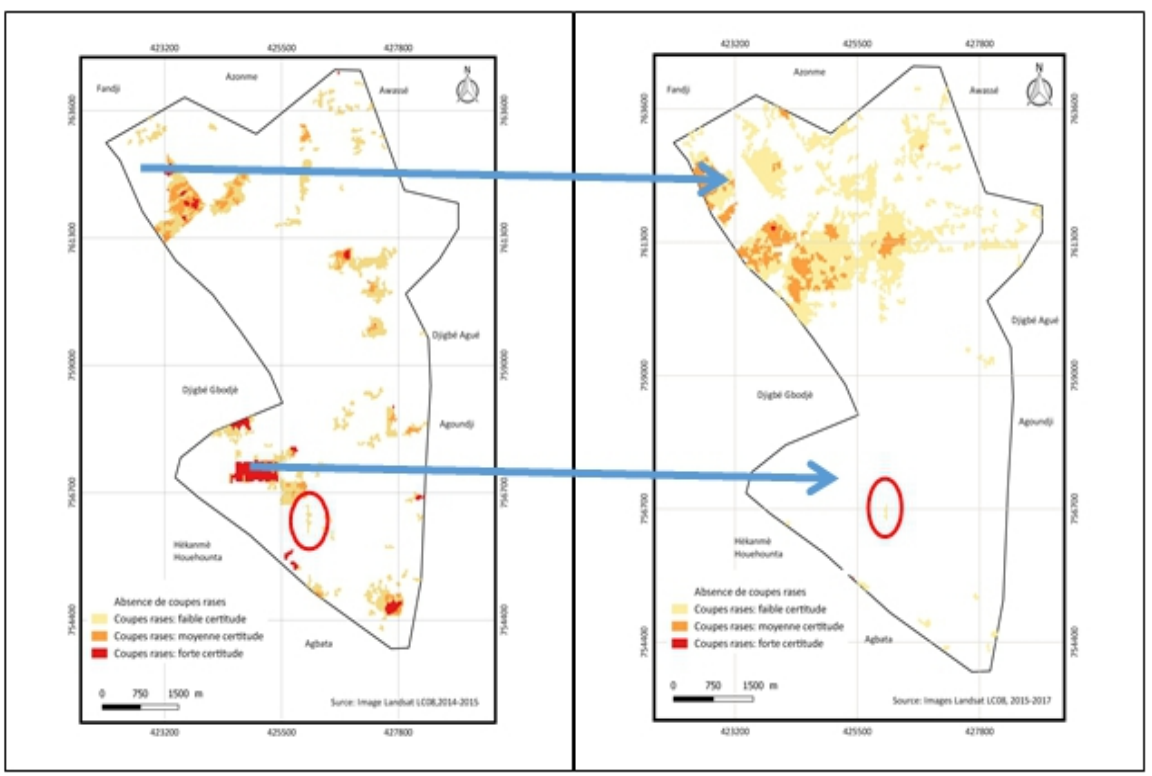

5. Répartition de coupes par degré de certitude (20142015)

5b. Répartition de coupes par degré de certitude (2015-2017)

Figure 5. Répartition des coupes-rases par degré de certitude, 2014-2015 et 2015-2017

\section{Répartition des coupes-rases par classe de superficie}

Il a été considéré les parcelles de coupes-rases supérieures à un hectare. Les superficies détectées pendant les périodes 2014-2015 et 20152017 sont respectivement de $13 \%$ et de $6 \%$. La Figure 6 présente les classes de répartition des 234,63 hectares de détections supérieures ou égales à 1 ha de décembre 2014 à janvier 2017. Au niveau de la classe de « 1 à 2 » ha il est détecté une superficie de 27,72 ha (13\%). Au niveau de la classe de « 2 à 3 » ha, il est détecté une superficie de 18,45 ha (9\%). Celle de « 3 à 10 » ha montre une détection de $37,08(18 \%)$ ha et celle supérieure à 10 ha présente une détection majoritaire de superficie de 127,62 ha (60\%) de coupes-rases. Il en résulte que la plupart des opérations de coupes-rases sont faites sur des superficies supérieures à 10 ha, révélant des blocs de grandes superficies coupées lors d'une campagne d'exploitation forestière. Par contre, très peu de coupes-rases semblent avoir été faites sur de petits espaces. Déterminer avec exactitude toutes les tailles surfaciques détectées pendant la période tiendrait probablement compte de tous les états de sols nus. Or la calibration de la détection réalisée sur une surface minimale de 1ha, aurait l'avantage de réduire les erreurs de commission. Il en est de même pour le choix de la calibration avec les degrés $2 \sigma$ et $3 \sigma$. 


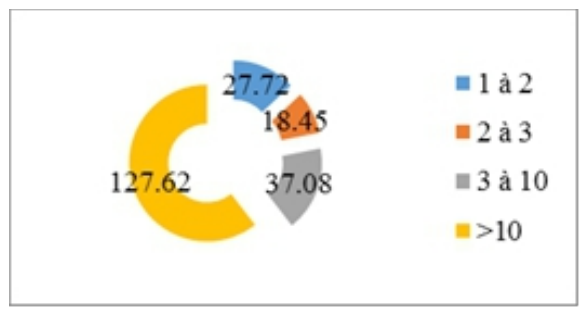

Figure 6. Répartition des coupes-rases de moyenne et forte certitude par classe de surface

L'intégration des cartes des coupes-rases successives de 2014 à 2017 de superficies supérieures ou égales à un (01) hectare a permis la réalisation d'une carte de l'ensemble de la période (Figure 7). Ces coupes-rases ont été détectées selon les degrés de certitude moyenne et forte. La surface totale des coupes-rases détectée pendant cette période est de 234,63 hectares (ha).179, 1 ha $(76 \%)$ sont de certitude moyenne et 55,53 ha (24\%) de certitude forte. Ces proportions révèlent les classes de probabilité des zones affectées de coupes-rases déterminé par la télédétection dans les plantations de Djigbé pendant toute la période considérée. Les coupes-rases sont majoritairement détectées vers le Nord-Ouest ; soit 154,28 ha de moyenne certitude et 9,81 ha de forte certitude. Vers le Sud, les coupes-rases sont moyennement détectées sur 24,55 ha de moyenne certitude et 43,92 ha de forte certitude. Vers le centre, les coupes-rases sont faiblement détectées sur 3,87 ha de moyenne certitude et 1,8 ha de forte certitude.

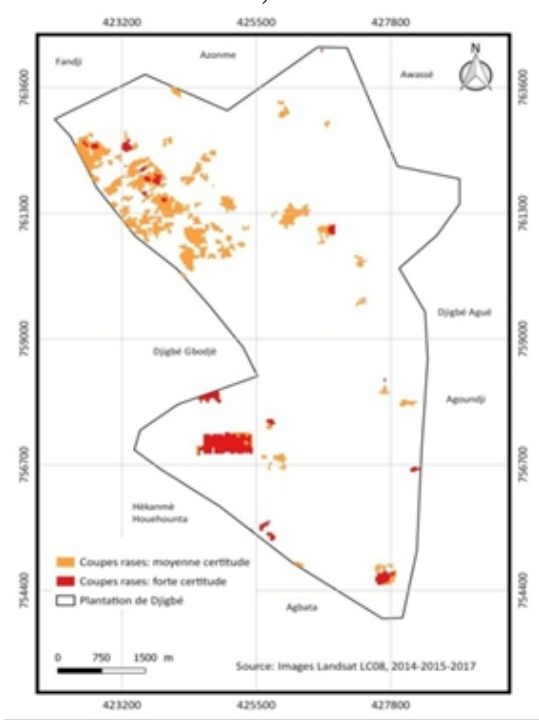

Figure 7. Détection globale des coupes-rases: moyenne et forte certitudes (20142017)

\section{Evolution des surfaces des coupes-rases}

L'évolution générale des surfaces de coupes-rases détectées de 2014 à 2017 sont présentées sur la Figure $8 \mathrm{a}$. La Figure $8 \mathrm{~b}$, quant à elle présente l'évolution à la même période, mais par degré de certitude. L'évolution 
générale révèle que les coupes-rases détectées de la période de décembre 2015 à janvier 2017 (67\%) ont presque été le double de celles détectées la période précédente (33\%). La Figure $8 \mathrm{~b}$ montre que de 2015 à 2017, les coupes-rases ont fortement régressées du faible degré de certitude $(80,3 \%)$ au fort degré de certitude $(0,4 \%)$. Une combinaison entre les périodes et les degrés de certitude, montre que pendant qu'il y a progression pour le degré1, les superficies des coupes-rases de degré 2 et 3 sont demeurées presque stables pendant la période 2014-2015 (115,74 ha) et 2015-2017(118,89 ha). Cette discordance dans la détection de l'évolution des superficies suivant le degré de certitude peut s'expliquer par le fait que la période 2015-2017 prend en compte le mois de janvier de l'année 2017 en plus. Ce mois de plus pourrait coïncider avec les moments de mis en défend de feu où les sols sont davantage mis à nu. A ce moment, il y a des opérations de passage d'un état végétatif à un état non végétatif. Ce qui pourrait apporter une confusion pendant le processus de détection des coupes-rases ou des interférences pendant les changements d'état de la végétation.

Figure 8. Evolution des superficies de coupes-rases par période et par degré de

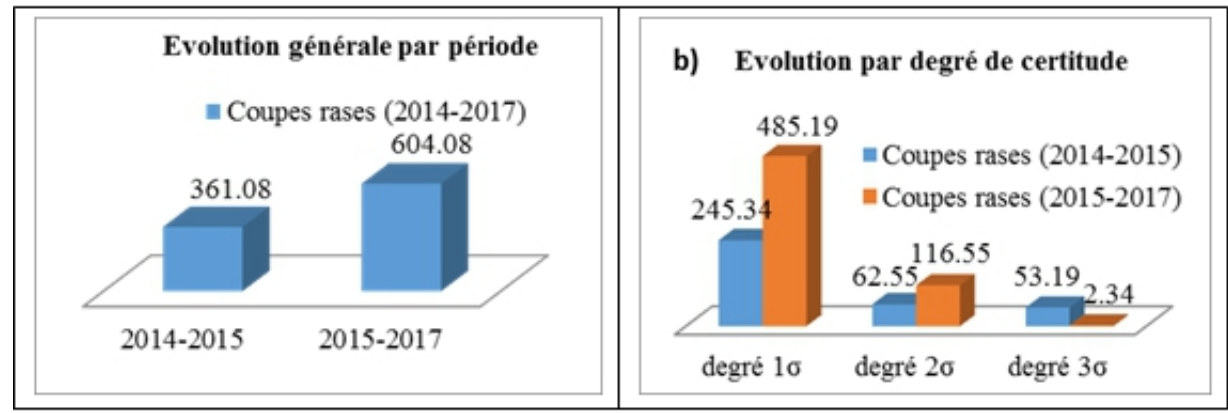

certitude.

\section{Evaluation statistique de la qualité du traitement}

Cette partie évalue la qualité de détection des coupes-rases au moyen des matrices de confusion.

Evaluation statistique par traitement d'image satellitaire avec ENVI (classification supervisée)

La matrice de confusion de la classification supervisée de l'image Landsat 8 de 2015 révèle que la précision globale de la qualité de la classification est de 93,93\% et que le coefficient de Kappa est égal à 87,86 $\%$ (Tableau I). Ceci illustre une bonne performance de la discrimination entre classe d'occupation du sol. 
Tableau I. Matrice de confusion de la classification supervisée de l'image Landsat 8 (2015), section plantation Djigbé.

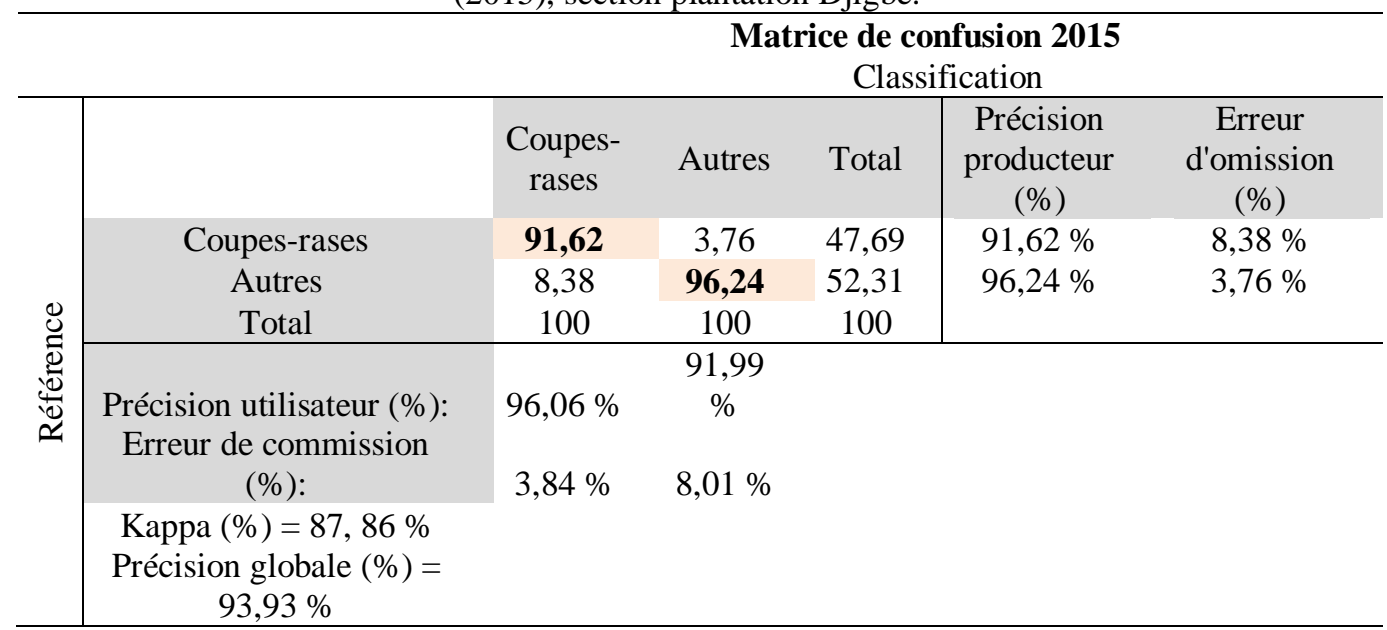

L'évaluation statistique de la détection des coupes-rases de la période 20152017 faite avec l'image Landsat 8 (2017) donne une matrice de confusion qui pourrait révéler que la classification est excellente $($ Kappa $=91 \%)$ avec une précision globale de 95,70\% (Tableau II). Lorsqu'on l'associe à la bonne connaissance du terrain.

Tableau II. Matrice de confusion de la classification de l'image Landsat 8 (2017), section plantation Djigbé

\begin{tabular}{l|lccc|cc}
\hline \multicolumn{7}{c}{ Matrice de confusion 2017 } \\
Classification
\end{tabular}

\section{Evaluation statistique avec QGIS utilisant les données de référence sur} le terrain

N'ayant pas eu suffisamment de données de référence sur le terrain pour la période 2014-2015, l'évaluation de la qualité de détection s'est 
limitée à la période 2015-2017 pour laquelle nous avons assez de données terrain de vérification (100 points de vérification).

. La validation des résultats pour la période 2015-2017 est réalisée à l'aide de la matrice de confusion mentionnant les erreurs d'omission et de commission. Les résultats de précision de la classification sont présentés au tableau III généré avec QGIS et Libre Office Calculator. Une centaine de polygones correspondant aux zones de coupes-rases sont identifiées. Les données obtenues ont généré le tableau de contingence.

Tableau III. Matrice de confusion de la classification supervisée de l'image Landsat 8 (2017) issue des données de terrain, section plantation Djigbé.

\begin{tabular}{cccccc}
\hline & 0 & 1 & Total & $\begin{array}{c}\text { Précision producteur } \\
(\%)\end{array}$ & $\begin{array}{c}\text { Erreur d'omission } \\
(\%)\end{array}$ \\
\hline 0 & 0 & 10 & 10 & 0 & 100 \\
1 & 0 & 90 & 90 & 100 & 0 \\
Total & 0 & 100 & 100 & & \\
\hline $\begin{array}{c}\text { Précision utilisateur } \\
(\%)\end{array}$ & $\mathrm{X}$ & 90 & & & \\
$\begin{array}{c}\text { Erreur de } \\
\text { commission (\%) }\end{array}$ & $\mathrm{X}$ & 10 & & & \\
Précision globale (\%) & & 90 & & & \\
\hline
\end{tabular}

Légende : $0=$ absence de coupes-rases ; 1 = présence de coupes-rases

\section{Discussion}

Cette étude a été réalisée afin de détecter et d'estimer les coupes-rases utilisant les images satellitaires Landsat suivant plusieurs dates. Les images Landsat TM, ETM+, OLI TIRS, sont beaucoup utilisées pour les études multi-dates afin d'apprécier l'évolution de la couverture du sol dans un espace donné. Plusieurs auteurs ont utilisé ces données comme Enonzan (2010), Mama (2013), Toko (2014), Kpedenou (2016), et Tente (2019). La détection des coupes-rases a été considérée pour des superficies supérieures ou égales à $900 \mathrm{~m}^{2}$. Guy G. et al. (2003), considèrent que cette estimation ne tenant pas compte des surfaces plus petite est inhérente aux caractéristiques de l'image Landsat et aux traitements morphologiques après classification qui ne conservent que les agrégats de plus 72 ares (8 pixels). En général, la précision de la détection de surfaces de coupes-rases dépend entre autres de la taille des surfaces impactées des peuplements (Fraser et al., 2005). Il a déjà été montré que les coupes de surface inférieure à 15 ha sont difficiles à détecter en utilisant les images MODIS (Bucha \& Stibig, 2008). La méthode basée sur des données MODIS a déjà toutefois montré son utilité pour détecter des dépérissement forestiers (Eklundh et al., 2009). Or la résolution de Landsat est largement supérieure à celle de MODIS. Ainsi donc, l'utilisation des images de type Landsat 8 de par sa résolution spatiale satisferait mieux à la taille de la zone d'étude. Cependant, si la méthode de coupes en des bandes alternées adoptée est telle que chaque bande à une 
dimension supérieure ou égale à $30 \mathrm{~m}$, les résultats seraient davantage améliorés. Les ombres de reliefs et les nuages constituent une limite à l'utilisation de l'outil (Ferrer, 2015). Or les images ne comportent pas de nuage au niveau de la zone d'étude. De ce fait, les résultats sont impactés positivement. La qualité de cette image ne peut être handicapante à la détection des coupes-rases de la période 2015-2017. La topographie du sol de la forêt de Djigbé n'est pas marquée de reliefs si élevé au point d'être un handicap aux résultats. Ce qui s'explique par les résultats intéressants de l'évaluation statistique. La sélection des images, à des dates assez proches (6 mois) pourrait optimiser davantage la détection. Ceci en rapport aux périodes considérées pour l'étude ; relativement longue pour laquelle il a été obtenu un résultat tout de même intéressant. Peu de données de référence étant disponibles pour des périodes rapprochées, le choix d'une période relativement longue satisfait aux vérifications statistiques. La date d'acquisition des images influence le NDVI et biaise la détection. En fonction de la date de prise de vue, le feuillage de la végétation peut être plus ou moins épais et impacter la valeur de NDVI. Les images acquises en début de feuillaison présentent un NDVI faible (Ferrer, 2015). Les périodes de feu de végétation peuvent aussi influencer les valeurs des NDVI. Les images utilisées dans la présente étude sont celles de fin de feuillaison et d'absence de feu de végétation dans la forêt de Djigbé. Cette forêt étant mise en défend et surveillée contre les feux de végétation, la possibilité d'une éventuelle influence des valeurs de NDVI liée au feu de végétation est à écarter.

L'évaluation statistique a concourue à l'obtention des résultats de bonnes qualités techniques et scientifiques. Les données de référence collectées sur le terrain et la photo-interprétation sur les images satellitaires traitées ont servis à vérifier la qualité des classifications. Les travaux de Ose et Deshayes (2015), utilisant la même approche ont donnés des résultats similaires. L'estimation des qualités de classification des travaux tel que ceux de (Toyi., 2012), (Mama, 2013), Ont abordé l'analyse des types d'occupation de sol ainsi que les facteurs de conversion d'un état a un autre. Les résultats des évaluations statistiques se basant sur des approches pareilles ont abouti à l'obtention des résultats aussi intéressants. En effet, l'évaluation statistique utilisant les données de références collectées sur le terrain a donné une précision globale de $90 \%$ et celle utilisant l'image satellitaire traitée par classification supervisée a donné une précision globale de 93,93\%. Dans le cadre de cette étude, il a été utilisé une méthode de détection des coupesrases selon le principe d'analyse de l'évolution de l'indice NDVI (Normalized Difference Vegetation Index ou indice de végétation normalisé) entre deux dates. C'est le plus largement utilisé. Cet indice présente son efficacité pour identifier les zones dégradées de végétation, plus spécialement dans les régions où la densité de végétation est forte et où la 
présence de sol est insignifiante (Bannari et al., 1996 ; Tchibozo \& Toundoh, 2014). La forêt classée de Djigbé présente des caractéristiques acceptables à l'utilisation de cet indice. Sensible aux variations spatiales et temporelles il est adapté pour le suivi de la dynamique de la végétation (Huete et al., 2002 ; Cheret et al., 2011). Ferrer (2015) a utilisé une approche similaire affirmant que cette méthode permet d'estimer de manière fiable le volume de bois coupés. Le résultat du traitement d'image à partir de NDVI conforte sur l'affirmation que le changement d'état de végétation à sol est perçu à partir des indices de végétation. Les résultats obtenus corroborent les travaux de ces auteurs en ce qui concerne l'efficacité des NDVI à détecter les changements d'état de végétation. Les spécialistes de la filière bois peuvent donc utiliser cette approche pour le suivi des coupes de grandes superficies par exemple. A l'instar de ce travail, Jin et Sader (2005), Bucha et Stibig (2008), ont consacré les leurs sur une classification binaire «changement pas de changements». L'avantage principal de ce type d'approche de la classification binaire, est que l'utilisateur final peut sélectionner le seuil de probabilité de passage en coupe selon ses objectifs, et surtout la précision attendue (Hansen et al., 2002). Pour affiner les résultats de la détection automatisée, nous avons regroupé en classes, la probabilité de présence de coupes par l'expression « degrés de certitude ». Le degré de certitude étant la probabilité de présence de coupes-rases. A l'instar d'Ose et Deshayes (2015) et Ferer (2015), il a été opté pour trois classes (faible, moyen et fort). Alors que Lambert (2014) a opté pour quatre classes (très faible, faible, moyenne et très fort) de probabilité de présence de coupes. L'objectif de travaux conditionne le choix du degré de certitude. Il est important de choisir le degré de certitude qui limite au maximum les erreurs de commission. Pour les résultats de ce travail, orienté vers la quantification des surfaces coupées, les degrés de certitude moyen $(2 \sigma)$ et fort $(3 \sigma)$ limitent au maximum les erreurs de commission. Ces résultats concordent avec ceux de Lambert (2014), Ferer (2015) ainsi que d'Ose et Deshayes (2015). Cheret et al. (2011), quant à eux ont défini les niveaux de dépérissement en les regroupant en classes. Ce qui a permis de quantifier globalement le phénomène de dépérissement des boisements résineux. Dans le cas de cette étude, le choix de probabilité appliqué au moyen et fort degré de certitude a permis de répondre à l'objectif de masquer les situations présentant un niveau de probabilité de présence de fausses coupes-rases élevées.

\section{Conclusion}

Cette étude a permis grâce à la télédétection et au SIG d'identifier les coupes-rases dans la forêt classée de Djigbé. Leur identification grâce à l'outil télédétection, a permis l'obtention des résultats pouvant permettre l'optimisation du contrôle et du suivi de la gestion durable des plantations et 
des forêts. Des coupes-rases avérées ont été ciblées et comparées avec des données de terrain et de documents de gestion forestière disponibles. Le choix des images doit être minutieux pour limiter les erreurs liées aux dates d'acquisition ou à la présence des nuages et d'ombres bien qu'il est possible de faire des traitements pour améliorer la netteté. Une grande attention doit aussi être apportée lors du processus de traitement afin d'éviter par exemple les erreurs d'inversement des bandes. L'utilisation des images de Landsat 8 du capteur OLI-TIRS a permis d'avoir des résultats satisfaisants d'après les vérifications statistiques. Une image de ce type à l'avantage de traiter de grandes surfaces assez rapidement, comparée aux images de plus haute résolution spatiale. La non disponibilité des images de haute résolution de la période d'étude et surtout leur coût d'acquisition sont des facteurs limitant à leur utilisation pour comparer leur résultat à celui de Landsat 8 utilisé dans cette étude. Cette méthode de cartographie et de télédétection des coupes rases doit être suffisamment déployée pour permettre le retour des utilisateurs. L'utilisation de la télédétection pour une détection annuelle ou mieux biannuelle des coupes-rases permettra aux opérateurs de la filière bois, de suivre et d'anticiper sur l'évolution du massif des plantations. Les données des résultats obtenus grâce à la télédétection et au SIG pourraient servir à la gestion durable des forêts par une approche de simulation prédictive. La télédétection et les SIG par cette méthode pourraient être utiles aux politiques dans le cadre de campagnes de reboisement. En effet, il pourrait être détecté dans des vastes forêts et plantations, des portions de zones non replantées après exploitation. La perspective d'une cartographie dynamique pour le suivi régulier et précis de nos forêts est un défi prometteur.

\section{References:}

1. Akouehou, G.S. \& Ayelo, G.H. (2005). De la conduite des peuplements issus de la régénération naturelle à la sylviculture : cas du teck (Tectona grandis) dans de la plantation domaniale de Djigbé au sud Bénin. Bulletin de la Recherche Agronomique du Bénin $\mathrm{N}^{\circ} 47-$ Mars 2005 : pp 7-18.

2. Aoudji, A.K.N. \& Ganglo, C.J. (2006). Phytosociologie appliquée à l'aménagement des forêts : cas du périmètre forestier de Pahou (Département de l'Atlantique, Sud-Bénin). J. Bot. Soc. Bot. France, 34, pp 89-92.

3. Aoudji, A.K.N., Ganglo, C.J., Adjakidjè, V., de Foucault, B. \& Azontondé, A.H. (2006). Phytocénose à Barteria nigritana Hook. f. et Rauvolfia vomitoria Afzel. dans le sous-bois des plantations de bois de feu de la forêt classée de Pahou, Sud-Bénin. J. Bot. Soc. Bot. France, 34, pp 81-88. 
4. Bannari, A., Huete, A.R., Morin, D. \& Zagolski, F. (1996). Effets de la Couleur et de la Brillance du sol sur les Indices de végétation, International Journal of Remote Sensing, n¹7(10): pp1885 -1906.

5. Bucha, T. \& Stibig, H.-J. (2008). Analysis of MODIS imagery for detection of clear cuts in the boreal forest in north-west Russia, Remote Sensing of Environment, 112 (5): pp 2416-2429.

6. Cheret, V., Denux Jean-Philippe, Gacherieu, C., \& Ortisset Jean-Pierre (2011). Utilisation de séries temporelles d'images satellitales pour cartographier le dépérissement des boisements résineux du Sud Massif Central. Rendez-vous Techniques ONF, pp.55-62. ffhal-01511003.

7. Crutzen, F. (2017). Approche multi-capteurs pour la cartographie par télédétection des ressources ligneuses en Wallonie : application à la commune de Paliseul. Faculté de Gembloux Agro-Bio Tech 68 p: URI/URL : http://hdl.handle.net/2268.2/2964.

8. Deshayes, M., Guyon, D., Jeanjean, H., Stach, N., Jolly, A., \& Hagolle, O. (2006). The contribution of remote sensing to the assessment of drought effects in forest ecosystems, Ann. For. Sci., vol. 63 pp.579595.

9. DGFRN (Direction Générale des Forêts et des Ressources Naturelles) (2010). Rapport d'activités 2009. Cotonou, Bénin, 20 p.

10. Dossa, O.S.N.L., Ganglo, C.J., Adjakidjè, V., Agbossou, E. \& de Foucault, B. (2005). Identification et caractérisation des stations forestières pour un aménagement durable de la forêt classée de Toffo (Département du plateau ; sud-est du Bénin). Bull. Rech. Agron. Bénin, 48, pp 48-59.

11. Enonzan, F.B. (2010). Utilisation de la télédétection et des SIG dans la gestion durable des aires protégées : cas des forêts classées de DogoKétou au Bénin. Mémoire de DESS en en Production et Gestion de l'Information Géographique, www.rectas.org, Campus universitaire Obafemi Owolowo, Ilé-Ifè, Nigéria, pp.49-50.

12. FAO (2003). La gestion des forêts tropicales secondaires en Afrique: réalité et perspectives. Rapport national du Bénin. Atelier FAO/EC LNV/GTZ. Douala, Cameroun, pp 17 - 21 novembre 2003.

13. Ferrer, F. (2015). Télédétection et cartographie des coupes-rases en Rhône-Alpes. Mémoire de master. Centre Agro Paristech. Université de Lorraine. $70 \mathrm{p}$

14. Furusawa, T., Pahari, K., Umezaki, M., \& Ohtsuka, R. (2004). Impacts of selective logging on New Georgia Island, Solomon Islands evaluated using very-high-resolution satellite (IKONOS) data. Environmental Conservation, 31 : pp 349-355. 
15. Fraser, R.H., Abuelgasim, A., \& Latifovic, R. (2005). A method for detecting large-scale forest cover change using coarse spatial resolution imagery, Remote Sensing of Environment, 95 (4): 414-427.

16. Ganglo, J.C. \& de Foucault, B. (2006). Plant communities, forest site identification in Toffo reserve, South-Benin. Bois Forêts Trop., 288 (2),pp 25-38.

17. Gond, V. \& Guitet, S. (2009). Élaboration d'un diagnostic postexploitation par télédétection spatiale pour la gestion des forêts de Guyane. Bois et Forêts des Tropiques, 2009, n 299 (1) : pp 5-13.

18. Guy, G., Gérôme, P., Nicolas, B. \& Thierry, B. (2003). Suivi de la gestion durable du massif aquitain de Pin Maritime par télédétection spatiale. Food and AgricultureOrganization of the United Nations. 0628-B1, http: // www.fao.org/docrep.article/wfc/XII.0628-B1.HTM.

19. Hansen, M.C., Defries, R.S., Townshend, J.R.G., Sohlberg, R., Dimiceli, C., \& Carroll, M. (2002). Towards an operational MODIS continuous field of percent tree cover algorithm: examples using AVHRR and MODIS data, Remote Sensing of Environment, 83(1-2), p.303-319.

20. Huete, A., Didan, K., Miura, T., Rodriguez, E.P., Gao, X., \& Ferreira, L.G. (2002). Overview of the radiometric and biophysical performance of the MODIS vegetation indices, Remote Sensing of Environment, vol.83, pp. 195-213

21. Jin, S. \& Sader, S.A. (2005). MODIS time-series imagery for forest disturbance detection and quantification of patch size effects, Remote Sensing of Environment, 99 (4), pp. 462-470.

22. Kpedenou, K.D., Boukpessi, T., Thiou Tanzidani, K., \& Tchamie, T.T.K. (2016). Quantification des changements de l'occupation du sol dans la préfecture de Yoto (sud Est Togo) a l'aide de l'imagerie satellitaire Landsat. Rev. Sc. Env. Univ., Lomé (Togo), 2016, n 13 pp.137-156.

23. Lambert, J.M. (2014). Evaluation des baisses de vitalité des peuplements forestiers à partir de séries temporelles d'images satellitaires, application aux résineux du sud du massif central et à la sapinière pyrénéenne. Thèse de doctorat, Ecole doctorale SEVAB, Université de Toulouse, 168p.

24. Mama, A. (2013). Anthropisation des paysages au Bénin: dynamique, fragmentation et développement agricole, Thèse de doctorat de ULB, $216 \mathrm{p}$.

25. Massim-Ouali, G. (2014). Foresterie communuataire et analyse de la contribution de l'exploitation des ressources-bois dans l'amélioration (durable) du bien -être des menages : cas de la forêt classée de Bassila, memoire de DEA, 64p 
26. MEPN (Ministère de l'Environnement et de la Protection de la Nature), PNUD (Programme des Nations Unies pour le Développement) (2009). Quatrième rapport national du Bénin sur la convention des nations unies sur la diversité biologique. p. 24. En ligne : http://www.cbd.int/doc/world/bj/bj-nr-04-fr.

27. Noumon, J.C. \& Ganglo, J.C. (2005). Phytosociologie appliquée à l'aménagement des forêts: cas du périmètre forestier de Koto (Dépatement du Zou, Centre Bénin). Acta Bot. Gallica, 152 (3), pp 421-426.

28. Noumon, C.J., Ganglo, C.J., Azontondé, A.H., de Foucault, B. \& Adjakidjè, V. (2006). Phytocénose à Mallotus oppositifolius (Geisl.) Müll. Arg et Deinbollia pinnata Schumach. \& Thonn. dans le sous-bois des teckeraies du Centre-Bénin. J. Bot. Soc. Bot. France, 36, pp 35-61.

29. ONAB (2005). Plan d'aménagement participatif des plantations forestières de Djigbé. Période 204-2023, 56p.

30. OIBT (2005). Critères et indicateurs révisés de l'OIBT pour l'aménagement durable des forêts tropicales, Modèle de rapport sur les C\&I inclus, Série OIBT: Politique forestière $\mathrm{n}^{\circ} 15$. Yokohama.

31. Ose, K. \& Deshayes-Irstea, M. (2015). Détection et cartographie des coupes-rases par télédétection satellitaire: guide méthodologique version 5.0 umrtetisirstea $72 \mathrm{p}$.

32. PNGDRN (2008). Programme National de Gestion Durable des Ressources Naturelles. Rapport Final, Novembre 2008. MEPN/DGRFN/ /GTZ 81p.

33. Quenum, C. (2005). Expansion du commerce intra et interrégionale entre les pays de la CEMAC et de l'UEMOA, Bénin. Étude de l'offre et de la demande sur le bois et ses produits dérivés. Observatoire des opportunités d'Affaires du Bénin (OBOPAF), $129 \mathrm{p}$

34. Read, J., Clark, D., Venticinque, E. \& Moreira, M. (2003). Application of merged 1-m and 4-m resolution satellite data to research and management in tropical forests. Journal of Applied Ecology, 40 : pp 592-600.

35. Solberg, S., Eklundh, L., Gjertsen, A.K., Johansson, T., Joyce, S., Lange, H., Naesset, E., Olsson, H., Pang, Y., \& Solberg, A. (2007). Testing remote sensing techniques for monitoring large scale insect defoliation, In Proceedings of the ForestSat'07 International Conference: spatial operational tools for forestry, Montpellier (France), 5p.

36. Souza, C., Firestone, L., Silva, L., \& Roberts, D. (2003). Mapping forest degradation in the Eastern Amazon from SPOT-4 through spectral mixture models. Remote Sensing of Environment, 87: pp 494506. 
37. Tchibozo, E. \& Toundoh, O. (2014). Détection localisée des zones dégradées de végétation par télédétection: application à la zone de transition entre le bassin sédimentaire et le socle cristallin (Centre Bénin). Innovative Space of Scientific Research Journals Vol. 11 No. 2: pp 477-493. http://www.ijisr.issr-journals.org/

38. Tente, O., Oloukoi, J., \& Toko, I. (2019). Dynamique spatiale et structure du paysage dans la commune de Zè, Bénin. Conférence OSFACO : Des images satellites pour la gestion durable des territoires en Afrique, Cotonou, Bénin. ffhal-02189544f

39. Tohngodo, B.C., Ganglo, J.C., Agbossou, K.E., de Foucault, B. \& Adjakidjè, V. (2006b). Caractéristiques structurales, identification et caractérisation des stations forestières de la forêt classée de Bonou (sud-est Bénin). Sciences \& Nature, 3 (1), pp 39-47.

40. Toko, I.N. (2014). Cartographie et modélisation de la dynamique des parcours naturels des troupeaux transhumants dans les communes de Banikoara et de Karimama (Nord-Bénin). Mémoire de DEA / FLASH / UAC, Bénin, $101 \mathrm{p}$.

41. Toyi, S.S.M. (2012). Analyse de l'impact de la diffusion du teck (Tectona grandis L.f.) sur la structure du paysage dans le Département de l'Atlantique (Sud-Bénin). Thèse de Doctorat unique en Sciences Agronomiques, Université d'Abomey-Calavi (Bénin), 216p.

42. Yêvidé, S.A., Ganglo, CJ., Aoudji, K.A., Toyi Sch, M., Charles de Cannière, Bruno de Foucault, Devineau Jean-Louis \& Sinsin, B. (2011). Caractéristiques structurelles et écologiques des phytocénoses de sous-bois des plantations privées de teck du département de l'Atlantique (Sud-Bénin, Afrique de l'Ouest), Acta Botanica Gallica, 158:2, 263-283, DOI: 10.1080/12538078.2011.10516272. 